\title{
Stenotrophomonas maltophilia as a Cause of Meningitis in an Infant
}

\author{
Arpita Shah ${ }^{1} \cdot$ Tanu Singhal $^{2}$ \\ Received: 2 March 2021 / Accepted: 3 May 2021 / Published online: 20 May 2021 \\ (C) Dr. K C Chaudhuri Foundation 2021
}

To the Editor: Stenotrophomonas maltophilia is a gramnegative rod ubiquitous in the environment and a cause of serious infections in hospitalized patients [1]. We report a case of community-acquired (CA) S. maltophilia meningitis in a healthy infant.

A 9-mo-old healthy infant presented with fever, vomiting, and irritability for $3 \mathrm{~d}$ and a bulging fontanelle. This was preceded a wk ago by an episode of gastroenteritis. Lumbar puncture was done and IV ceftriaxone initiated. Hb was $7.5 \mathrm{~g} \%$ and TLC was $8100 / \mu \mathrm{L}$. The CSF showed 11,200 cells, $90 \%$ polymorphs, protein $272 \mathrm{mg} \%$, and sugar $13 \mathrm{mg} \%$ (blood sugar 78). The fever resolved initially to reappear after 48 h. The CSF culture grew $S$. maltophilia, sensitive to ceftazidime, levofloxacin, and cotrimoxazole (VITEK 2). The isolate was considered a contaminant and LP repeated as fever was persisting. Repeat CSF showed 500 cells, 75\% lymphocytes, protein $111 \mathrm{mg} \%$, and sugar $55 \mathrm{mg} \%$ (blood sugar 80). Repeat CSF culture sent to a reference laboratory again grew S. maltophilia (MALDI TOF MS) with same sensitivity. Treatment was changed to intravenous cotrimoxazole and levofloxacin following which, fever resolved and CSF normalized. Antibiotics were stopped after $2 \mathrm{wk}$. The child is normal over a follow-up period of $18 \mathrm{mo}$.

CA infections due to $S$. maltophilia are uncommon and occur in those with comorbidities and the immunocompromised $[1,2]$. There is only one published case of CA meningitis in a healthy term neonate [3]. Contaminated water seems to be the most likely source of infection in our case [4]. While this case is a rarity, it emphasizes the need for reconfirmation of diagnosis by repeat testing when rare or unusual pathogens are cultured and the patient is not improving despite appropriate empiric therapy. Occurrence of second rare infection in such a patient should also trigger investigation for an immunodeficiency disorder.

Data Availability Case details available, if required.

\section{Declarations}

Conflict of Interest None.

\section{References}

1. Singhal L, Kaur P, Gautam V. Stenotrophomonas maltophilia: from trivial to grievous. Indian J Med Microbiol. 2017;35:469-79.

2. Khanum I, Ilyas A, Ali F. Stenotrophomonas maltophilia meningitis - a case series and review of the literature. Cureus. 2020;12:e11221.

3. Ibrahim J, Hamwi N, Rabei H, Abdelghafar M, Al-Dulaimi Z, Al TH. Stenotrophomonas maltophilia meningitis in a term healthy neonate: a case report and literature review. Case Rep Pediatr. 2018;2018:1543934.

4. Verweij PE, Meis JF, Christmann V, et al. Nosocomial outbreak of colonization and infection with Stenotrophomonas maltophilia in preterm infants associated with contaminated tap water. Epidemiol Infect. 1998;120:251-6.

Publisher's Note Springer Nature remains neutral with regard to jurisdictional claims in published maps and institutional affiliations.
Tanu Singhal

tanusinghal@yahoo.com

1 Department of Pediatrics, Khatav Mother \& Child Hospital, Mumbai, Maharashtra, India

2 Department of Pediatrics \& Infectious Disease, Kokilaben Dhirubhai Ambani Hospital and Medical Research Institute, Mumbai, Maharashtra 400053, India 